\section{Are rates of vision loss in patients in English glaucoma clinics slowing down over time? Trends from a decade of data}

Eye (2015) 29, 1639; doi:10.1038/eye.2015.186

Correction to: Eye (2015) 29, 1613-1619; doi:10.1038/ eye.2015.161; published online 28 August 2015

Since the online publication of the above article, the authors have noted some errors.

In the abstract, results section, the following sentence has been edited as follows:

'Median rate of MD loss in older (> 70 years) eyes was faster than that observed in younger $(<60$ years) eyes $(-0.21$ compared with $-0.01 \mathrm{~dB} /$ year $).$

Figure 1 and the legend have been edited. The corrected figure and legend is shown below.
T Boodhna, LJ Saunders and DP Crabb

The summary text, What this study adds, has also been replaced with the following text:

Average visual field progression rates have only improved marginally over the last decade. The proportion of fast progressors has not reduced. Visual fields of older eyes are, on average, found to progress faster than younger eyes. There is no evidence of patients being stratified to receive more or less frequent visual field examination, given their age, severity of glaucoma, or progression speed.

The authors apologise for any inconvenience caused by these errors.

These errors have now been rectified, and the corrected article appears in this issue. The html and online pdf versions have also been rectified, and now carry the corrected paper.

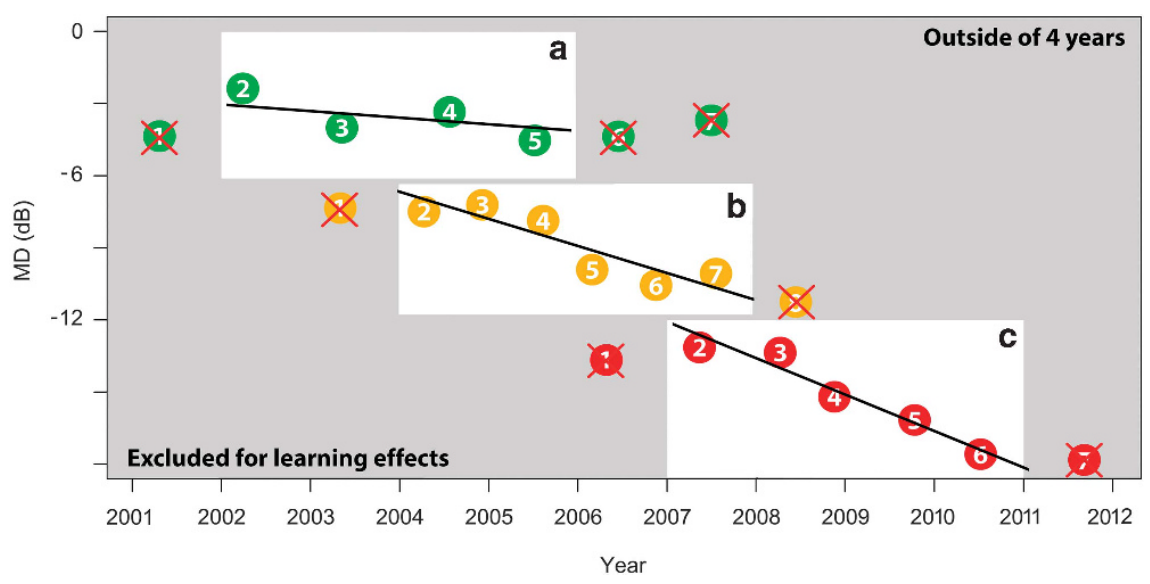

Figure 1 A schematic illustrating the VF series inclusion criteria and method for calculating rates of MD loss (dB/ year) for three example eyes detected in 2001 (a), 2003 (b), and 2006 (c). Eyes were excluded for o5 VF examinations or $\mathrm{o} 4$ years of follow-up. The first VF in each series was omitted to account for perimetric learning effects. Rate was calculated from linear regression of the baseline VF and the series of exams that fell within a 4-year period after it (white window). So, for example, for series (a) the sixth and seventh recorded VFs fall outside this window and are not used in the calculation. This ensures that all rates are estimated with equivalent precision, allowing for comparisons over time. A minimum of three VFs were required to be in this 4-year window. This rate was then assigned to the date of the baseline exam. 